“C 2005 IEEE. Personal use of this material is permitted. Permission from IEEE must be obtained for all other uses, in any current or future media, including reprinting/republishing this material for advertising or promotional purposes, creating new collective works, for resale or redistribution to servers or lists, or reuse of any copyrighted component of this work in other works." 


\title{
A 3D Vector Magnetization Model with Interaction Field
}

\author{
Jin Jiang ZHONG, Jian Guo ZHU, Senior Member, IEEE, YouGuang GUO, Member, IEEE,
} and Zhi Wei LIN

\begin{abstract}
This paper presents a vector model of magnetization based on the 3D Stoner-Wohlfarth elemental operator. To account for the magnetic interactions between particles, a phenomenological mean-field approximation is employed. The paper also illustrates the numerical simulation results of the magnetization in 3D. This model will be useful to simulate the magnetization process of complicated topology flux electromagnetic devices.
\end{abstract}

Index Terms-Magnetic moment, magnetization, 3D S-W elemental operator, mean field, isotropic and anisotropic material.

\section{INTRODUCTION}

A lthough a lot of research, both theoretical and experimental, has been conducted to explain the magnetization process in magnetic materials, it is still not fully understood yet. In general, the magnetic field strength is vectorial, and the magnetization process in magnetic materials has to be considered using vector magnetization. Thus, the vector magnetization $\mathbf{M}$ of materials must be treated as a vector with magnitude and direction.

There are at least four approaches to the modeling of vector magnetic hysteresis. The first approach is statistically analogous to the one dimensional Preisach model [1-8] in which the particle interactions are introduced through a distribution function. However, it is not particularly useful for gaining a physical understanding. In addition, it is criticized on the grounds that it fails to properly describe the rotational properties of magnetic materials. This is partly because of the questionable assumptions used in coupling the vector hysteresis components, and the fact that the Preisach model does not include reversible magnetization.

The second approach is the Stoner-Wohlfarth (S-W) model [9-17]. This model can describe both reversible and irreversible magnetization processes, and are easier to be practiced under rotating fields, for example, it is commonly applied to simulation of thin film material and powder magnets. Despite the fact that it is inherently a three dimensional vector model, not many papers studying the behavior of S-W model in three dimensions (3D) have been presented in the literature.

The third model is the combined model [18-24]. Theoretically, this model incorporates the vector elemental

Manuscript received June 8, 2004.

The authors are with the Center for Electrical Machine and Power Electronics, Faculty of Engineering, University of Technology, Sydney, NSW 2007 Australia (telephone: +61-2-95141268, e-mail: zhong@eng.uts.edu.au, joe@eng.uts.edu.au,youguang@eng.uts.edu.au, jacklin@eng.uts.edu.au). operator of the S-W model into the Preisach diagram such that the new model has the vector nature of the $S-W$ model while retaining the efficiency of the Preisach model. The main problem with this model is that the identification of the distribution function $P(\psi, \xi, \alpha, \beta)$ by fitting the model to experimental data has not been adequately addressed. In addition, the design of the combined model does not address the question of what part of the input history leaves its mark upon the future output of vector hysteresis, which causes difficulties for numerical implementation of the model.

The fourth approach is the microscopic model [25-26] in which the details of particle interactions are taken into account. However, the microscopic computation requires too much computing power, and there is still a significant discrepancy between the model and experiment.

This paper presents a vector model of magnetization based on the 3D S-W elemental operator. This model assumes that a magnetic material consists of an infinite number of single domain acicular particles with uniaxial anisotropy. To account for the magnetic interactions between particles, a phenomenological mean-field approximation is employed. This model provides a compromise between accuracy and computational convenience, is useful to simulate the magnetization process of complicated topology flux electromagnetic devices.

\section{3D S-W ELEMENTARY OPERATOR}

Considering a single domain particle in three dimensional space, the $\mathrm{X}$ axis is chosen as the easy axis of the particle. When a magnetic field applied, the magnetic moment $\mathbf{m}_{\mathbf{s}}$ of the particle rotates to the orientation which results in minimum energy, as shown in Fig.1.

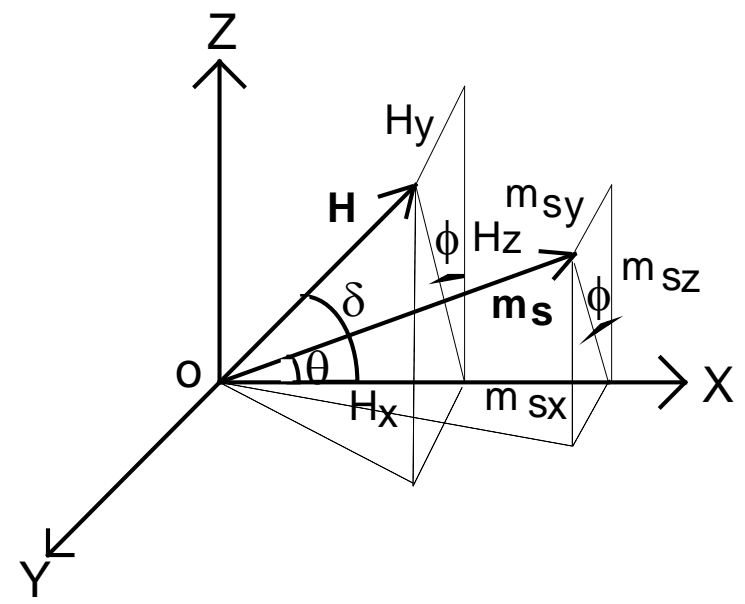

Fig. 1. Rotation of $\mathbf{m}_{\mathbf{s}}$ under $\mathbf{H}$ in $3 \mathrm{D}$ 
The total free energy of a single domain with moment $\mathbf{m}_{\mathbf{s}}$ is

$$
E(\theta, \mathbf{H})=K \sin ^{2} \theta-\mu_{0} \mathbf{m}_{\mathrm{s}} \bullet \mathbf{H}
$$

The positions of minimum energy can be found by solving the equation $\partial E(\theta, \mathbf{H}) / \partial \theta=0$ for $\partial^{2} E(\theta, \mathbf{H}) / \partial \theta^{2}>0$. It can be shown that there are two energy minima if a small field is applied. As the field strength increases, the positions of these minima change. Initially, these changes are reversible. When the applied field strength exceeds a certain critical value $H_{c}$, however, one of the energy minima becomes unstable, and the magnetic moment of domain jumps to the other minimum, which is the global energy minimum. This critical point at which the irreversible domain rotation occurs is the point of minimum energy for which $\partial^{2} E(\theta, \mathbf{H}) / \partial \theta^{2}=0$, finally we have

$$
H_{x}^{2 / 3}+\left(H_{y} \sin \phi+H_{z} \cos \phi\right)^{2 / 3}=H_{k}^{2 / 3}
$$

where $H_{k}=2 \mathrm{~K} / \mu_{0} m_{s}$, is the crystal anisotropy field, $\phi$ is the angle between the plane $\mathrm{XOZ}$ and the plane $\mathrm{XOH}$ that contains the magnetic field $\mathbf{H}$ and the easy axis of particle, $\phi$ varies over 360 degree. Equation (2) expresses a 3D S-W elemental operator, as illustrated in Fig.2

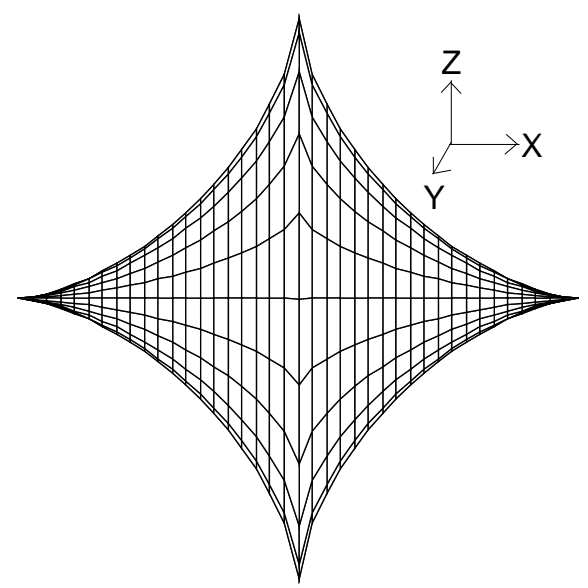

Fig. 2. 3D S-W elemental operator

$$
\begin{aligned}
& \text { For a specified field } \mathbf{H}\left(\mathrm{H}_{\mathrm{x}}, \mathrm{H}_{\mathrm{y}}, \mathrm{H}_{\mathrm{z}}\right) \text {, } \\
& \phi=\tan ^{-1}\left(H_{y} / H_{z}\right) \text {. Thus, the current equilibrium orientation } \\
& \text { of } \mathbf{m}_{\mathrm{s}} \text { can be evaluated by means of the asteroidal rule in the } \\
& \text { plane XOH. The equilibrium direction of } \mathbf{m}_{\mathrm{s}} \text { in space is finally } \\
& \text { determined by } m_{x}=m_{s} \cos \theta, \quad m_{y}=m_{s} \sin \theta \sin \phi \text {, } \\
& m_{z}=m_{s} \sin \theta \cos \phi .
\end{aligned}
$$

\section{Magnetization With MEAn Field}

In the S-W model, the interaction field between the particles is ignored. However, for many materials, the interaction effect between particles is important and cannot be ignored.
The interaction field can be precisely computed by using the micro-magnetic models [25-26]. However, this requires huge computing power. To achieve a compromise between the computational accuracy and speed of the model, Jiles and Atherton [27] postulated and used a mean field interaction $\mathbf{H}_{\mathrm{m}}=\alpha \mathbf{M}$, which expresses a means of approximating the actual interactions [14-17]. The mean field is a good choice to account for the macroscopic effect of the interaction between the $\mathrm{S}-\mathrm{W}$ particles. Thus, the total effective field is

$$
\mathbf{H}_{e}=\mathbf{H}_{a}+\alpha \mathbf{M}
$$

where $\alpha$ is a dimensionless mean field parameter for isotropic materials, which theoretically can be identified from experimental data [27], $\mathbf{H}_{\mathbf{e}}$ the total effective field and $\mathbf{H}_{\mathbf{a}}$ the applied external field.

Sequentially, the magnetization $\mathbf{M}$ for bulk materials is the vector sum of contributions of all the constituent particles, and can be mathematically expressed as

$$
\mathbf{M}=S\left(\mathbf{H}_{a}+\alpha \mathbf{M}\right)
$$

where $S($ ) stands for the S-W model.

In numerical implementation, the magnetization is obtained by the vector sum of the magnetic moments $\mathbf{m}_{\mathbf{s}}$ of an assembly of $\mathrm{N}_{\mathrm{p}}$ magnetic particles, as expressed below

$$
\mathbf{M}=\sum_{i=1}^{N_{p}} \mathbf{m}_{\mathbf{s i}}\left(\mathbf{H}_{a}+\alpha \mathbf{M}\right)
$$

\section{NUMERICAL SiMULATIONS}

The simulations for the magnetization processes of an isotropic magnetic material under both alternating and rotating magnetic fields have been carried out. It is implemented by numerical algorithm in MATLAB. Fig.3(a) and (b) show the reversible and jump processes of the magnetic moment $\mathbf{m}_{\mathbf{s}}$ of a single magnetic particle under alternating and circular rotating excitation fields $\mathbf{H}$ respectively in space. The stars “*” and plus "+" represent the loci $\mathbf{m}_{\mathbf{s}}$ and $\mathbf{H}$ respectively.

In another simulation, $1800 \mathrm{~S}-\mathrm{W}$ pseudo-particles are assumed to be uniformly distributed in a plane. The vector magnetization $\mathbf{M}$ is computed under an alternating field. Fig.4(a) illustrates the hysteresis loop of $\mathrm{M}_{h}-\mathrm{H}$, where $\mathrm{M}_{\mathrm{h}}$ is the component of magnetization aligned with the external field. Since the particles are uniformly distributed, all other components of $\mathbf{M}$ are zero, and the vector magnetization $\mathbf{M}$ is equal to $\mathbf{M}_{\mathrm{h}}$. This means the alternating field $\mathbf{H}$ and magnetization $\mathbf{M}$ are collinear in the symmetric case. In a magnetically anisotropic material, the S-W particles are not uniformly distributed, and the magnetization $\mathbf{M}$ will not be aligned with the applied field $\mathbf{H}$. The position of magnetization $\mathbf{M}$ is thus determined by both the field strength $\mathbf{H}$ and the particle distribution function. 


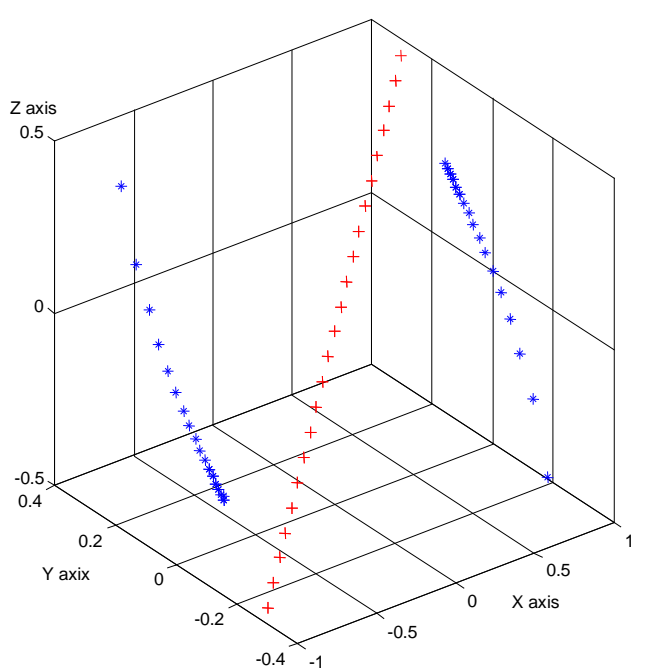

(a)

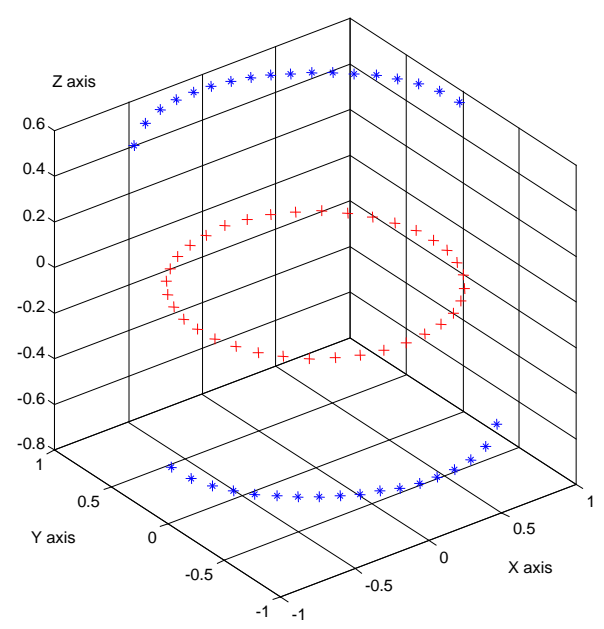

(b)

Fig. 3. $\mathbf{m}_{\mathbf{s}}$ loci of an S-W single domain particle with (a) alternating $\mathbf{H}$, and (b) rotating $\mathbf{H}$

Similarly, 508 magnetic particles are uniformly distributed in three dimensions. The magnetic particles of material are homogeneously arranged in the ray cone. Fig.4(b) illustrates the loci of the rotating magnetic field strength $\mathbf{H}$ and the magnetization $\mathbf{M}$ of material. A circular rotating field is applied on the plane that is inclined $30^{\circ}$ from the horizontal plane. According to the calculation, the $\mathbf{M}$ components perpendicular to the circular rotating field plane are cancelled due to the uniform distribution of the particles. Thus, the magnetic magnetization $\mathbf{M}$ is in the same plane with the field strength $\mathbf{H}$. The calculation shows that for a normalized field $\mathbf{H}$ of 0.6 , the magnitude of the normalized $\mathbf{M}$ is 0.82 , and which lags the excitation field by about $10^{\circ}$. While in a magnetically anisotropic material, the magnetic magnetization $\mathbf{M}$ is not in the same plane of the rotating field strength $\mathbf{H}$. As mentioned above, the position of magnetization $\mathbf{M}$ is determined by the field strength $\mathbf{H}$ and the particle distribution function.

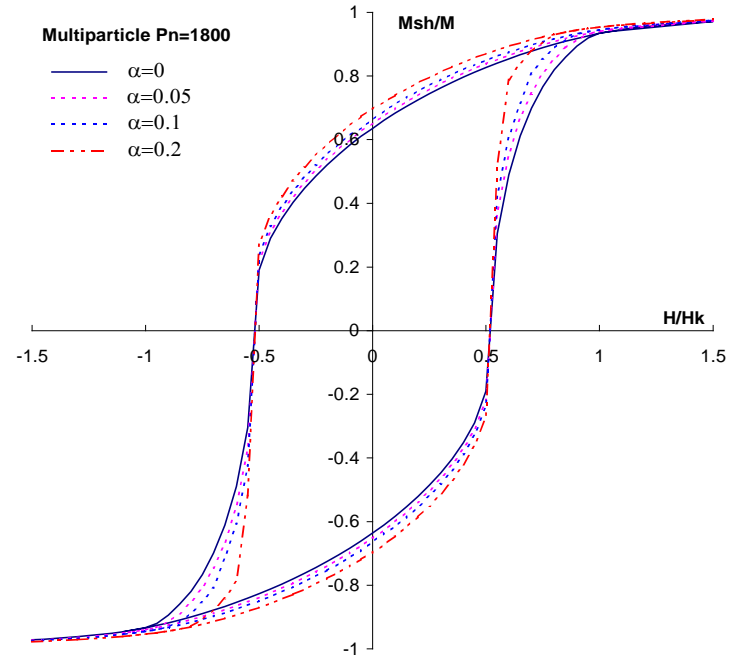

(a)

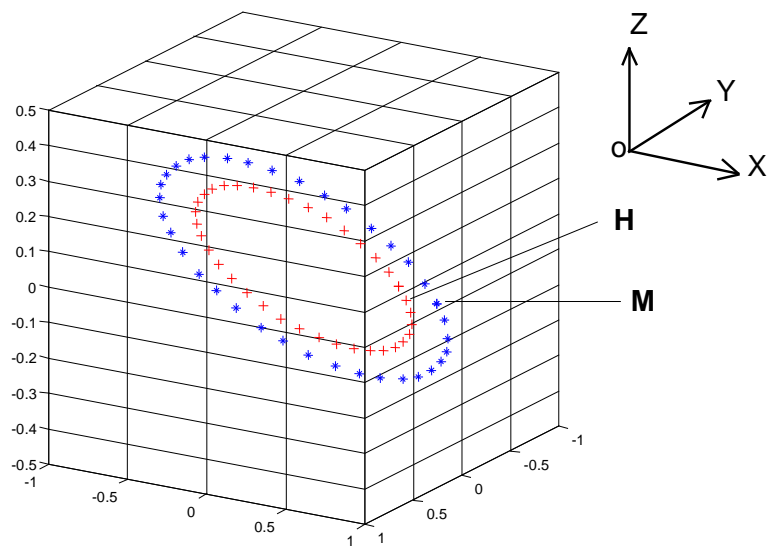

(b)

Fig. 4. (a) hysteresis loop of $\mathbf{M}-\mathbf{H}$, and (b) $\mathbf{M}$ loci with rotating $\mathbf{H}$

\section{CONCLUSION}

The vector magnetization of multi-particles under either an alternating excitation field or a rotating excitation field is evaluated by using the modified S-W model which incorporates a mean interaction field into the S-W elemental operator. The results show that in an isotropic magnetic material the magnetization vector and the magnetic field strength vector are collinear for an alternating magnetic field excitation. With a circular rotating magnetic field excitation the magnetization vector lags the magnetic field for a certain angle, and is in the same plane with the field. This model is useful to simulate the magnetization process of complicated topology flux electromagnetic devices.

\section{REFERENCES}

[1] E.D. Torre, J. Oti, and G. Kadar, "Preisach modelling and Reversible magnetization,” IEEE Trans. Magn., vol. 26, pp. 3052-3058, Nov. 1990.

[2] I.D. Mayergoyz, Mathematical models of hysteresis. Springer-Verlag, 1991

[3] K. Wiesen and S.H. Charap, "Vector Preisach modelling," J. Appl. Phys., vol. 61, pp. 4019-4021, Apr. 1987.

[4] E.D. Torre, G. Kadar, "Vector Preisach and the moving model," $J$. Appl. Phys., vol. 63, pp. 3004-3006, Apr. 1988. 
[5] F. Vajda, J. Oti, and E.D. Torre, "Rotational properties of vector phenomenological models of magnetic media,” J. Appl. Phys., vol. 67, pp. 5376-5378, May 1990.

[6] F. Vajda and E.D. Torre, "Experimental analysis of vector Preisach modeling for anisotropic recording media," IEEE Trans. Magn., vol. 31, pp. 2907-2909, Nov. 1995.

[7] K.C. Wiesen and S.H. Charap, "A rotational vector Preisach model for unoriented media,” J. Appl. Phys., vol. 67, pp. 5367-5369, May 1990.

[8] A.A. Adly and I.D. Mayergoyz, "A new vector Preisach-type model of hysteresis,” J. Appl. Phys., vol. 73, pp. 5824-5826, May 1993.

[9] E.C. Stoner, F.R.S. and E.P. Wohlfarth, "A mechanism of magnetic hysteresis in heterogeneous alloys,” Phil. Trans. Roy. Soc., vol. 240A, pp. 599-642, May 1948.

[10] I.B. Ortenburger and R.I. Potter, "A self-consistent calculation of the transition zone in thick particulate recording media,” J. Appl. Phys., vol. 50, pp. 2393-2395, Mar. 1979.

[11] I.A. Beardsley, "Self-consistent recording model for perpendicularly oriented media,” J. Appl. Phys., vol. 53, pp. 2582-2584, Mar. 1982.

[12] T.R. Koehler, "A computationally fast, two dimensional vector hysteresis model,” J. Appl. Phys., vol. 61, pp. 1568-1575, Feb. 1987.

[13] J. Oti and E.D. Torre, "Efficient numerical model of magnetic switching of fine particles with uniaxial anisotropy," IEEE Trans. Magn., vol. 27, pp. 3908-3911, Sept. 1991.

[14] D.L. Atherton, and J.R. Beattie, "A mean field Stoner-Wohlfarth hysteresis model,” IEEE Trans. Magn., vol. 26, pp. 3059-3063, Nov. 1990.

[15] A.L. Ribeiro, "Magnetic hysteresis model for magnetic recording including spatial fluctuations of interaction fields," J. Appl. Phys., vol. 69, pp. 4841-4843, Apr. 1991.

[16] J.S. Yang, C.R. Chang, and I. Klik, "A causality algorthm for magnetic hysteresis of interacting systems," IEEE Trans. Magn., vol. 32, pp. 1120-1123, May 1996.
[17] Luca Callegaro and Ezio Puppin, "Rotational hysteresis model for stressed ferromagnetic films,” IEEE Trans. Magn., vol. 33, pp. 10071011, Mar. 1997.

[18] J. Oti and E.D. Torre, "A vector moving model of both reversible and irreversible magnetizing processes,” J. Appl. Phys., vol. 67, pp. 53645366, May 1990.

[19] H.A.J. Cramer, "A moving Preisach vector hysteresis model for magnetic materials," J. Magnetism Magn. Mater., vol. 88, pp. 194204, 1990

[20] S.H. Charap and A. Ktena, "Vector preisach modeling,” J.Appl. Phys., vol. 73, pp. 5818-5823, May 1993.

[21] F. Ossart, R. Davidson and S.H. Charap, "Moving vector Preisach model and $\delta \mathrm{M}$ curves," IEEE Trans. Magn., vol. 30, pp. 4360-4262, Nov. 1994.

[22] F. Ossart, R. Davidson and S.H. Charap, "A 3D moving vector Preisach Hysteresis model,” IEEE Trans. Magn., vol. 31, pp. 17851788, May 1995.

[23] R.J. Davidson and S.H. Charap, "Combined vector hysteresis models and applications," IEEE Trans. Magn., vol. 32, pp. 4198-4203, Sep. 1996.

[24] C.S. Koh, S.Y Hahn, and G.S Park, "Vector hysteresis modeling by combining Stoner-Wohlfarth and Preisach models," IEEE Trans. Magn., vol. 36, pp. 1254-1257, July 2000.

[25] J.G. Zhu and H.N. Bertram, "Micromagnetic studies of thin metallic films,” J. Appl. Phys., vol. 63, pp. 3248-3253, Apr. 1988.

[26] W. Williams and D.J. Dunlop, "Three dimensional micromagnetic modeling of ferromagnetic domain structure," Nature, vol. 337, pp. 634-637, Feb. 1989.

[27] D.C. Jiles and D.L. Atherton, "Theory of ferromagnetic hysteresis," J. Magnetism Magn. Mater., vol. 61, pp. 48-60, 1986. 